\title{
Robotic Interaction with Deformable Objects under Vision and Tactile Guidance - a Review
}

\author{
Fouad F. Khalil and Pierre Payeur \\ School of Information Technology and Engineering, University of Ottawa \\ Ottawa, ON, Canada, K1N 6N5 \\ [fkhalil, ppayeur]@site.uottawa.ca
}

\begin{abstract}
This paper overviews the modern concepts adopted by the robotics community during the past decade related to $3 D$ deformable object grasping and manipulation mainly guided by vision and force/tactile sensing. It also examines the new trends on rigid objects manipulation which can be extended to deformable objects. This constitutes a challenging area of research because of the complexity of interactions between the robot manipulator and the deformable object due to its deformation. To this effect, there are many issues to be considered in order to develop a truly autonomous robotic system that can successfully manipulate $3 D$ deformable objects. This investigation concludes by proposing viable solutions for designing an intelligent robotic framework to manipulate $3 D$ deformable objects without any human intervention.
\end{abstract}

Keywords - 3D deformable object manipulation, autonomous robots, force/tactile probing, dexterous grasping, computer vision.

\section{INTRODUCTION}

Modern robotic systems used in industrial, security and space applications require a new generation of autonomous robot manipulators [1] able to perform intelligently sophisticated manipulation tasks in environments which may be relatively unknown, variable or unstructured. Therefore, much research efforts have been devoted within the past decade on the development of robots that can sense and react to their environment and to the type of object to be handled, whether rigid or deformable. If robots can act intelligently based on sensed information, the need for specialized well calibrated workspaces can be reduced as robots become more tolerant in dealing with unknown objects. To build such autonomous systems requires the combination of instrumentation, control systems, computer and mechanical engineering.

Future autonomous robotic systems aiming at real world applications in manufacturing, space, or in the field of security require more flexible and general solutions carried out by imitating the basic human vision-touch capabilities. Human experience with objects manipulation provides an existing evidence of the ability of vision to assist in grasping and manipulation tasks. Autonomous manipulators have to produce similar coordination between these two sensory systems to work efficiently in unknown environments and adapt to unpredictable modifications. Vision provides rich knowledge about the spatial arrangements, that is, the geometry and topology of objects to be manipulated. The grasping process can be improved by using vision feedback such that the robot end-effector can be guided by the vision system and hence the relationship between the object and the end-effector can be estimated. However, integrating the visual feedback with contact/force sensing can cope with the inaccuracy of vision systems alone due to problems like occlusion and inability of the vision sensor to provide force estimation.

For the major part of robotics literature, however, the objects to be grasped and manipulated are considered to be rigid. Less effort has been made in investigating the manipulation of deformable objects. But in reality, numerous objects are of such type. That is, they are mostly unsymmetrical, compliant, and have alterable shapes. Robot manipulation of deformable objects still offers an important challenge to the robotics community and makes it a subject of significance for the development of the future generation of autonomous robots.

Aside from reviewing the main issues and trends encountered when dealing with rigid objects manipulation $[2,3,4,5]$, which can be extended to deformable objects in a sensor based robotic setup, this review focuses on recent solutions introduced for deformable objects manipulation [6-23]. The objective is to categorize the generic problem of deformable objects manipulation into more specific sub-problems, mainly corresponding to the modeling and simulation of deformable objects, the fusion of measurements taken from vision and force/contact sensors, and the required control scheme to handle the manipulation process. This leads to the proposal of a design framework for the development of an autonomous robotic system to manipulate 3D deformable objects under vision and tactile guidance, which is the objective of our current research effort.

\section{ROBOTIC INTERACTION}

Typically in a robotic setup, when robot manipulators are programmed to perform their tasks, they must have a complete knowledge about the exact structure of the manipulated object (shape, surface texture, rigidity) and about its location in the environment (pose). For some of these tasks, the manipulator becomes in contact with the object. Hence, interaction forces and moments are developed and consequently these interaction forces and moments, as well as the position of the end-effector, must be controlled, which leads to the concept of "force controlled manipulation" [2]. There are two basic control strategies used in general to solve the force controlled manipulation problem and to develop the required contact task control with the environment. These are respectively the hybrid position/force control scheme [24] and the impedance control scheme [25].

Alternatively, to enhance interaction with the object, the process of measuring the variables resulting from the application of the forces on the object must be considered. Another important aspect of interaction with the object is the derivation 
of a contact model and the selection of proper grasp points [26, 27]. These have a crucial role in performing the grasping process where the objective is always to mimic the human hand [28]. To achieve the resemblance with human arm/hand in robotics, force/tactile sensors [29] can be mounted on robotic hands that are usually comprised of two or more fingers. These types of sensors give crucial information such as the presence of a contact with the object, its size and shape, the exchanged forces between the object and the robot hand, mechanical properties of the object in contact, and detection of slippage of the body in contact. A smart combination of all this information opens the door to more sophisticated manipulation known as dexterous manipulation [30]. Hand dexterity refers to the capability of changing the position and orientation of the manipulated object from a given reference configuration to a different one arbitrarily chosen within the hand workspace. It is a rather broad concept that involves aspects of ability and stability in performing motions of the manipulated object by hand palm and fingers.

\section{HANDLING RIGID OBJECTS}

There exists a substantial research effort carried out in the literature on robotic grasping and manipulation of rigid objects where deriving the contact and grasping model is one of the basic operations in the manipulation process. Form closure and force closure are the most widely covered topics on grasp modeling that concerns the conditions under which a grasp can restrain an object. These two concepts have been originally proposed for evaluating stable grasping of rigid objects. Form closure grasp [31] considers the placement of frictionless contact points so as to fully restrain an object. Alternatively, force closure grasp [32] is more related to the ability of a grasp to reject disturbance forces and usually considers frictional forces. On the other hand, power grasps [33] are characterized by multiple points of contact between the grasped object and the surfaces of the fingers and palm and hence increase grasp stability and maximize the load capability.

In another category, the research on multifingered robot grasping modeling can be classified as fingertip grasp and enveloping grasp [34] respectively. In fingertip grasp the manipulation of an object is expected to be dexterous since the finger can exert an arbitrary contact force onto the object. On the other hand, when an object is grasped using the enveloping grasp model the grasping process is expected to be stable and robust against an external disturbance since the fingers contact with the object at many points. Bicchi and Kumar [26] and Mason [27] published extensive surveys on robot grasping and contact with rigid objects reviewing the concepts and methodologies used. There has been significant work in the robotics community as well towards recovering good grasp point candidates on the object. Watanabe and Yoshikawa [35] present a comprehensive review and different classifications for the methods used to choose such grasp points. In their work, choosing optimal grasp points was investigated on an arbitrary shaped object in $3 \mathrm{D}$ space using the concept of required external force set.

However, grasping and manipulation processes are carried out by controlling interaction forces at the contact points with the object. Hybrid position/force control [24] tries to decouple the directions in which force is controlled, e.g. the force normal to the surface, from those in which position is to be tracked, e.g. forces along the surface. In contrast to hybrid position/force control, the impedance control scheme [25] combines the position and force control rather than separating them and aims at softening the rigidity of robotic manipulators by assigning desired impedance to the end-effector. Using hybrid position/force control for controlling dexterous hands was investigated in [36] and impedance control in [37].

On the other hand, if interaction control is to be considered as a sensor-based problem, then two categories of research efforts can be distinguished. Force sensing is considered in the first category such that the manipulator can sense the interaction with the object during the interaction execution phase [2]. In this case force sensors can be used effectively to perform hybrid force/position control or impedance control strategies. However, force sensing can provide full 3D information only about the local contact with the grasped object. Alternatively, a vision system, which is considered in the second category [38], can produce the global information about the $3 \mathrm{D}$ environment. In this case the interaction control comes in form of visual feedback, which is often referred to as "visual servoing", to enable the robot to see the object and refine accordingly the interacting process. Unfortunately, vision approaches are not suitable to establish and maintain contact with the object surface if precise position and orientation are unknown.

Another trend emerged following some attempts to combine the two different complementary sensory systems, vision and force. A research effort was reported by Xiao et al. [3] on using hybrid position/force control with force sensor and a fixed camera. A sensor fusion strategy was proposed to merge force/torque-based and vision-based measurements. A combined vision/force control scheme was also reported by Baeten and De Schutter [4] using both force and vision sensors mounted on the end-effector at the same time. Using this eye-in-hand camera configuration, a common global 3D framework for both force and vision control was proposed to model, implement and execute robotic tasks in an uncalibrated workspace. The method to control the orientation of the end-effector using the force/torque sensor in this framework was investigated later by Zhang et al. [5] and it was found that the torque measurement is not accurate enough for a free-form surface, which could cause orientation control errors. As a result, this method appears not to be appropriate for feature tracking with accurate orientation control on an unknown surface. To overcome this problem an automated robot path generation method was developed in [5] based on vision, force and position sensor fusion in an eye-inhand camera configuration that is used to identify the line or edge feature on a free form surface. A robot is then controlled to follow the feature more accurately.

\section{MANIPULATION OF DEFORMABLE OBJECTS}

The main challenge in developing autonomous robotic systems to manipulate deformable objects comes from the fact that there are different generic interconnected problems to be solved. It involves the collection of deformation characteristics, the modeling and simulation of the deformable object from these estimates, and the definition and tuning of an efficient control scheme to handle the manipulation process based on multisensory feedback. A recent trend aims at merging measurements taken from vision and force/ tactile sensors to 
accelerate the development of autonomous robotic systems capable of executing intelligent exploratory actions and to perform dexterous grasping and manipulation.

\section{A. Deformable Objects Modeling and Simulation}

Automatic handling of deformable objects usually requires that the evaluation of the deformation characteristics is carried out using simulated environments before conducting the physical experiment. Hence, the manipulation process can be successfully performed by analyzing the manipulative tasks and deriving their control strategies using deformable object models. A wide variety of approaches have been presented in the literature dealing with computer simulation of deformable objects [6]. These approaches are mainly derived from physically-based models that emulate physical laws to produce physically valid behaviors. Mass-spring system simulations and finite elements methods are the major physically-based modeling techniques considered [39].

In addition to computer modeling and simulation of deformable objects, other research efforts in robotics were dedicated to the modeling problem in the process of the physical manipulation. Nowadays, an important goal is to achieve stable grasp and manipulation of objects whose attributes and deformation characteristics are not known a priori. In fact, deformable objects change their shapes during manipulation and display a wide range of responses to applied interaction forces because of their different physical properties. This is due to their nonlinearity attributes and other uncertainties. In order to implement and evaluate the manipulative operations on deformable objects by a robotic system, an object model is indispensable to represent the elasticity and deformation characteristics during the physical interaction. For this purpose, the modeling problem for 1D and 2D deformable objects was studied extensively as in [7, 8], based on mathematical representations of their internal physical behavior. In one of the recent efforts, a finite element modeling technique was reported in [9] where a model is developed to control the grasping and manipulation of a deformable object based on internal force requirements. In this model the object deformation is related to fingertip force, and based on impedance control of the endeffector.

Modeling of 3D deformable objects has not been widely addressed in literature so far. This results from its complexity and the fact that a majority of researchers hope to tackle the simpler 1D modeling problem before generalizing to a $3 \mathrm{D}$ modeling solution. Among the very few research efforts on $3 \mathrm{D}$ modeling is the pioneering work reported by Howard and Bekey [10] who developed a generalized solution to model and handle 3D unknown deformable objects. The model used to represent the viscoelastic behavior is derived from dividing the object into a network of interconnected particles and springs according to Kelvin model. Then by using Newtonian equations, the particle motion is used to calculate the deformation characteristics based on neural networks. Other interesting methods for modeling 3D deformable objects are based on probing the object to extract the deformation characteristics with the aid of vision. One of these methods was developed in [11] to acquire deformable models of elastic objects in an interactive simulation environment where an integrated robotic facility was designed to probe the deformable object in order to acquire measurements of interactions with the object. Another method of probing and vision tracking was reported in [12] to model deformable objects geometric and elastic properties. The approach uses vision and neural networks to select only a few relevant sampling points on the surface of the object and guides the acquisition of deformation characteristics through tactile probing on these selected points. The measurements are combined to accurately represent the 3D deformable object in terms of shape and elastic behavior.

Regarding the contact and grasp modeling for deformable objects, concepts of rigid force and form closure as well power grasp were extended to accommodate deformable objects. In [13], the concept of force closure for rigid objects with unbounded applied forces was extended to deformable objects with bounded applied forces. Wakamtatsu et al. introduced the concept of bounded force closure, which is defined as grasps that can resist any external force within the bound. They considered a candidate grasp and external forces within a bound that can deform and displace the deformed part. In [14], Prattichizzo et al. focus on the dynamics of the deformable objects during the process of power grasp. A geometric approach is adopted to derive a control law decoupling the internal force control action from the object dynamics. More recently, a new framework for grasping of deformable parts in assembly lines was proposed by Gopalakrishnan and Goldberg [15] based on form closure for grasping deformable parts. In this framework a measure of grasp quality is defined based on balancing the potential energy needed to release the part against the potential energy that would result in plastic deformation.

\section{B. Robotic Interaction Control with Deformable Objects}

In early robotic systems designed to manipulate deformable objects, the problem of interaction control was solved mainly in two different ways. The robotic system to handle deformable object was either designed based on force and grasp stability control [16], or force control versus deformation control [17]. Mandal and Payandeh [16] proposed a control strategy based on PID control to maintain stable contact against a compliant 1D surface. Meer and Rock [17] used impedance control to manipulate flexible objects in 2D. Other attempts were reported, such as the work of Shimoga and Goldenberg [18], to design systems with force control based on grasping with soft fingers. Overall, these systems require explicit models of the object which include in-depth knowledge about mass/object dynamics and deformability, and therefore, complex force sensory system is required to measure the position and force of the object. Recent developments by Foresti and Pellegrino [19] focus on automating the way of handling deformable objects using vision techniques only. Their vision system worked along with a hierarchical self-organizing neural network to select proper grasping points in $2 \mathrm{D}$.

However, dexterous grasping and manipulation of a deformable object must be performed robustly despite uncertainties in the robot environment where deformable objects are neither exactly located nor modeled. This leads to higher flexibility, and can improve speed and precision of the task execution. A number of recent research efforts focus on 
improving both the task quality and its range of feasibility by using integrated vision and force based control schemes. In such dexterous manipulation it is important to consider the difference between the way of handling rigid and deformable objects. Among other things, this leads to a major distinction between the definitions of grasping and manipulation respectively [20]. While the manipulation of a rigid object requires only the control of its location, the manipulation of a deformable object requires controlling both the location of the object and its deformation. In the handling process of rigid objects, grasping and manipulation can be performed independently. Grasping of a rigid object requires the control of grasping forces only, while manipulation of a freely moving rigid object results in the change of its position and orientation. On the other hand, grasping and manipulation interfere with each other in the handling of deformable objects. Grasping forces yield the deformation of a deformable object, which may change the shape and location of the object. Hence contact between fingers and the object may be lost and grasping may be compromised due to the deformation at the fingertips. Therefore, in the handling of deformable objects, grasping and manipulation must be performed in a collaborative way.

\section{Interaction under Combined Vision and Tactile Sensing}

The way of automating robotic manipulators to handle deformable objects in an unknown configuration typically involves an initial exploratory action by vision sensors to guide the robot arm toward the object, then visual information must be complemented by force/tactile measurements collected when a tactile probe or a dexterous hand comes in contact with the surface of the object. This supplementary data refines the knowledge about the position and orientation of the object and can provide an estimate of its elasticity or viscosity characteristics. All available information must be merged into a coherent model in order to allow the tuning of the feedback control loop that will guide the dexterous grasping and manipulation processes. Finally, tactile probing should continue during the operation using tactile sensors mounted at the fingertips to provide the necessary tactile sensitivity and sufficient dexterity to perform skillful manipulations of the deformable objects which may be of irregular shape and composition. Furthermore, visually monitoring of the task provides the necessary feedback to gauge how well the manipulator performed the task, or if an error has occurred, such as slippage.

In recent efforts to solve this dual interaction control problem, Hirai et al. [20] developed a robust control law for manipulation of $2 \mathrm{D}$ deformable parts using tactile and vision feedback to control the motion of the deformable object with respect to the position of selected reference points. Following this positioning approach, multiple points on a deformable object are guided to the final position. Another control method to cope with 2D deformable objects was proposed by Huang et al. [21] that relies on high speed visual detection and visual/force sensor fusion. In an extension to their initial work [22], they also proposed a stereovision based compliance control that enables a robot arm to manipulate an unknown deformable object. In a later study [23], a position/force hybrid control method that incorporates visual information with force control was introduced to enable a robot arm with a flexible tool in a form of a hose to perform the contact process with the unknown 2D deformable object.

\section{DEXTEROUS END-EFFECTORS}

Dexterous end-effectors in form of multifingered gripper or anthropomorphic hand have crucial role to support manipulation of deformable objects. In order to meet the requirements imposed by the interaction with deformable objects, dexterous hands must have a sufficient number of degrees of freedom and be equipped with tactile sensors on their fingertips and palm. A wide variety of dexterous robotic grippers/hands, both for technical use and as well as for humanoid robots, are already available to researchers, and some even to commercial applications. References [40] and [41] present some of the available state-of-the-art dexterous hands summarizing their features and specifications. Examples of the commercially available state-of-the-art dexterous hands include the Shadow Hand [42], the DLR-HIT Hand [43], and the Barrett Hand [44] that have the potential to be mounted on a suitable manipulator to develop an autonomous system for the purpose of handling deformable objects. However, there are still important issues that arise from the use and integration of such dexterous hands with off-the-shelf robotic manipulators. The main one comes from the fact that most robot arms commercially available do not offer an open architecture. Many newly developed dexterous hands also suffer from the same limitation, while their architecture, both mechanical and electronics, may not be suitable for a large range of applications.

Beyond laboratory experiments, these dexterous hands nowadays are expected to perform a wide range of applications in complex scenarios [45] and hence more sophisticated modeling and control schemes can be achieved using their extended sensor information. However, the modeling and control problem of dexterous hand has been solved in the literature in general using simplified approaches based on heuristic rules inferred from practical task execution. This is because of the mechanical model complexity and system nonlinearity which makes the optimization problem mathematically difficult to solve. The development of fundamental optimized dexterous control method is explored by Yin et al. [36] within the framework of hybrid control scheme. Biagiotti et al. [37] solved the problem based on impedance control with less mathematical constraints.

\section{PROPOSED FRAMEWORK}

It is noticeable that most of the control methods are applied to manipulate 1D and 2D deformable objects. The study of how to control a robot arm to handle a 3D deformable object still remains an open subject. An early attempt to produce generalized approach for handling 3D deformable objects was reported by Howard and Bekey [10] using force sensors and stereoscopic vision. Their work was to learn robotic grasping characteristics for deformable objects using neural networks by establishing a relationship between grasping force and material deformability in order to produce firm grasping forces. As a result, this work mainly addressed the modeling problem of 3D deformable objects without attempting to solve the control problem. Furthermore, the manipulation process conducted did not involve any dexterity considerations. Howard and Bekey 
investigated a collaborative grasping process that consisted of pushing against an object from two opposite ends using two cooperative manipulators to lift the object vertically against the gravity.

In our current research work, a framework is being developed that combines vision and tactile sensing to autonomously handle 3D deformable objects. The approach builds upon previous research on 3D probabilistic modeling [46] to combine robotic range sensing and tactile/force probing. The vision data is obtained from a range sensor to locate the object in an unknown environment and to guide the robot arm in proximity to the object. Then a dexterous hand/gripper provides measurements to determine the unknown object elasticity and deformation characteristics, and hence forces/ moments for grasping are calculated accordingly. The force/tactile sensors mounted on the robot arm and hand have a crucial role to refine the pose/orientation of the hand/gripper and to ensure stability while performing the grasping process. It is expected that fusing vision/touch information in the feedback loop can provide the necessary control law to allow on-line grasp planning and dexterous manipulation in a more efficient and effective way relaxing the time constraint spent on off-line learning, as in [10], while overcoming the limitations of stereoscopic vision. The combination of visual feedback and force/tactile probing can provide the necessary dexterity as well as the compliance and robustness required to grasp and manipulate deformable objects. This also coincides with the dexterity and robustness requirements reported in [20] and extended to the 3D case, without the need for extensive analysis and detailed knowledge about the object's elasticity characteristics.

The modeling scheme used in this framework is based on the development of enhanced 3D uncertainty maps generated by combining visual data with the object deformation characteristics collected by touch/probing under visual guidance. This scheme strictly relies on experimental measurements and does not imply any physical model of deformation characteristics. It provides all necessary information about the relationship between the endeffector and the deformable object. As such, the proposed framework can be considered a $3 \mathrm{D}$ extension of the algorithms in $[21,22,23]$ although conducted in a different manner. The point in common is that these algorithms can also perform the manipulation without the need for a physical model.

From an experimental point of view, the framework is implemented in a sequential manner comprised of three stages carried out in an automated setup, as shown in Fig. 1. At the first stage, a pre-grasping process of the object is performed to guide the arm toward the deformable object using range vision sensor and a probabilistic map indicating the location of the object in the environment. This map is first segmented using an original segmentation scheme introduced by Abou Merhy et al. [47]. As a second stage, the end-effector is brought in close proximity to the object to be manipulated. When the robot hand is in reach of the object, a preliminarily tactile probing process is initiated by taking measurements on selected sampling points [12] from force/tactile sensors mounted on the robot arm and end-effector to give further details about the object's elastic behavior. This information can be collected via multiple sensors, including a 6DOF force/torque sensor mounted at the robot's tool plate, and a set of force sensitive tactile pads installed on the fingertips of the robot gripper [48]. Merging the data from these various sources of information, an enhanced 3D model of the deformable object is computed that provides the object's characteristics about its size, shape, elasticity and deformation behavior. In the final stage, the grasping and manipulation processes are performed. The grasping step is controlled by an efficient control scheme that uses tactile force feedback to refine the gripper fingertips closure and enable safe and stable manipulation of the deformable object by the robot arm.

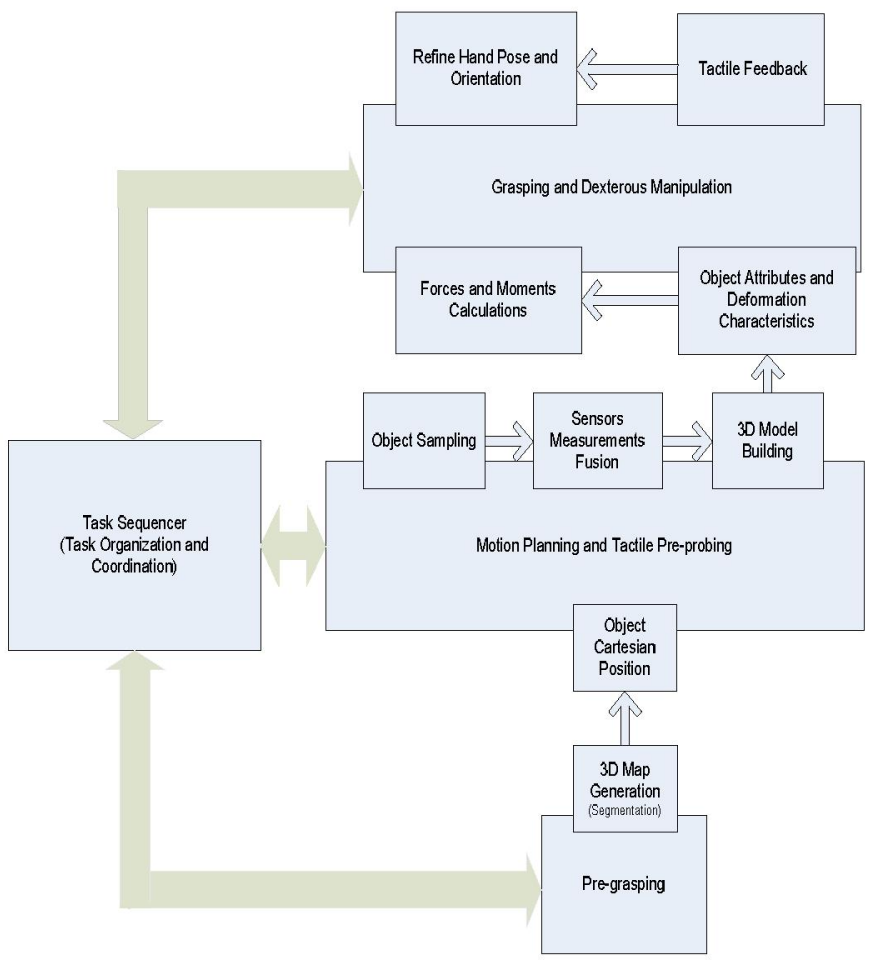

Fig 1. Block diagram of the proposed framework.

\section{CONCLUSION}

This paper reviews the major trends adopted in autonomous robotic interaction mainly guided by vision and force/tactile sensing over the last ten years. The review focuses on issues that are related or can be extended to 3D deformable objects manipulation. These aspects are essential to establish a novel framework for the purpose of dexterous handling of 3D deformable objects. The proposed framework builds upon an enhanced 3D probabilistic representation of deformable objects where visual information is combined with deformation characteristics obtained via selective tactile probing. The objective is to evolve the work of Howard and Bekey [10] by incorporating the control problem along with the modeling aspect in order to solve the grasping and manipulation tasks on 3D deformable objects.

\section{REFERENCES}

[1] M. Seitz, "Towards Autonomous Robotic Servicing: Using an Integrated Hand-arm-eye System for Manipulating Unknown Objects", J. of Robotics and Autonomous Systems, vol. 26, pp. 23-42, 1999. 
[2] C. Natale, "Interaction Control of Robot Manipulators: Six-degrees-ofFreedom Tasks", Springer Tracts in Advanced Robotics, vol. 3, Springer, Berlin, 2003.

[3] D. Xiao, B. K. Ghosh, N. Xi, and T. J. Tarn, "Sensor-Based Hybrid Position/Force Control of a Robot Manipulator in an Uncalibrated Environment", IEEE Trans. on Control Systems Technology, vol. 8, pp. $634645,2000$.

[4] J. Baeten and J. De Schutter, "Integrated Visual Servoing and Force Control - The Task Frame Approach", Springer Tracts in Advanced Robotics, vol. 8, B. Siciliano, O. Khatib, and F. Groen, Eds., Springer, Berlin, 2003.

[5] H. Zhang, H. Chen, and N. Xi, "Automated Robot Programming Based on Sensor Fusion", Int. J. of Industrial Robot, vol. 33, pp. 451-459, 2006.

[6] S. F. Gibson and B. Mirtich, A Survey of Deformable Modeling in Computer Graphics, MERL Technical Report, TR97-19, 1997.

[7] D. Henrich and H. Worn, Eds., Robot Manipulation of Deformable Objects, Springer-Verlag, London, 2000.

[8] M. Saadat and P. Nan, "Industrial Applications of Automatic Manipulation of Flexible Materials", Int. J. of Industrial Robots, vol. 29, pp. 434-442, 2002.

[9] S. Garg and A. Dutta, "Grasping and Manipulation of Deformable Objects Based on Internal Force Requirements", Int. J. of Advanced Robotic Systems, vol. 3, pp. 107-114, 2006.

[10] A. H. Howard and G. Bekey, "Intelligent Learning for Deformable Object Manipulation", J. Autonomous Robots, vol. 9, pp. 51-58, 2000.

[11] J. Lang, D. Pai , and R. Woodham, "Acquisition of Elastic Models for Interactive Simulation", Int. J. of Robotics Research, vol. 21, pp. 713$733,2002$.

[12] A. -M. Cretu, E.M. Petriu, and P. Payeur, "Neural Network Mapping and Clustering of Elastic Behavior from Tactile and Range Imaging for Virtualized Reality Applications", Proc. IEEE Int. Workshop on Imaging Systems and Techniques, pp. 17-22, 2006.

[13] H. Wakamtatsu, S. Hirai, and K. Iwata, "Static Analysis of Deformable Object Grasping Based on Bounded Force Closure", Proc. IEEE Int. Conf. on Robotics and Automation, pp. 3324-3329, 1996.

[14] D. Prattichizzo, P. Mercorelli, A. Bicchi, and A. Vicino, "On the Geometric Control of Internal Forces in Power Grasps", Proc. IEEE Conf. on Decision and Control, pp. 1942-1947, 1997.

[15] K. Gopalakrishnan and K. Goldberg, "D-Space and Deform Closure Grasps of Deformable Parts", Int. J. of Robotics Research, vol. 24, pp. 889-910, 2005.

[16] N. Mandal and S. Payandeh, "Control Strategies for Robotic Contact Tasks: An Experimental Study", J. Robotic Syst., vol. 12, pp. 67-92, 1995.

[17] D. W. Meer and S. M. Rock, "Experiments in Object Impedance Control for Flexible Objects", Proc. Int. Conf. Robotics and Automation, pp. 1222-1227, 1994.

[18] K. B. Shimoga and A. A. Goldenberg, "Soft Robotics Fingertips I: A Comparison of Construction Materials", Int. J. of Robotics Research, vol. 15 , pp. 320-334, 1996.

[19] G. L. Foresti and F. A. Pellegrino, "Automatic Visual Recognition of Deformable Objects for Grasping and Manipulation", IEEE Trans. on Systems, Man, and Cybernetics: Applications and Reviews, vol. 34, pp. 325-333, 2004.

[20] S. Hirai, T. Tsuboi, and T. Wada, "Robust Grasping Manipulation of Deformable Objects", Proc. IEEE Symp. on Assembly and Task Planning, pp. 411-416, 2001.

[21] J. Huang and I. Todo, "Control of a Robot Based on Fusion of Visual and Force/Torque Sensor Information (Manipulation of a Deformable Object)", Trans. Japan. Soc. Mech. Eng., vol. 67, pp. 2616-2623, 2001.

[22] J. Huang, I. Todo, and I. Muramatsu, "Neuro-control of a Robot Using Visual and Force/Torque Sensor Information (Manipulation of a Flexible Beam Object)", Trans. Japan. Soc. Mech. Eng., vol. 69, pp.2085-2092, 2003.

[23] J. Huang, I. Todo, and T. Yabuta, "Position/Force Hybrid Control of a Manipulator with a Flexible Tool Using Visual and Force Information", Cutting Edge Robotics, V. Kordic, A. Lazinica, and M. Merdan, Eds., pp. 611-628, ARS/plV, Germany, 2005.
[24] M. H. Raibert and J. J. Craig, "Hybrid Position/Force Control of Manipulators", ASME J. of Dynamic Sys. Meas. Control, vol. 102, pp. 126-132, 1981.

[25] N. Hogan, "Stable Execution of Contact Tasks Using Impedance Control", Proc. IEEE Int. Conf. on Robotics and Automation, pp. 1047$1054,1987$.

[26] A. Bicchi and V. Kumar, "Robotic Grasping and Contact: A Review", Proc. of IEEE Conf. on Robotics and Automation, pp. 348-353, 2000.

[27] M. T. Mason, "Mechanics of Robotic Manipulation", The MIT Press, 2001.

[28] K. Kaneko, K. Harada, and F. Kanehiro, "Development of MultiFingered Hand for Life-size Humanoid Robots", Proc. of IEEE Int. Conf. on Robotics and Automation, pp. 913-920, 2007.

[29] D. Javad and S. Najarian, "Advances in Tactile Sensors Design/Manufacturing and its Impact on Robotics Applications - A Review", Int. J. of Industrial Robots, vol. 32, pp. 268-281, 2005.

[30] A. Bicchi, "Hands for Dexterous Manipulation and Robust grasping: A Difficult Road Towards Simplicity", IEEE Trans. on Robotics and Automation, vol. 16, pp. 652-662, 2000.

[31] A. Bicchi, "On the Closure Properties of Robotics Grasping", Int. J. of Robotics Research, pp. 319-334, 1995.

[32] V. D. Nguyen, "Constructing Force-closure Grasps", Int. J. of Robotics Research, vol. 7, pp. 3-16, 1988.

[33] K. Mirza and D. E. Orin, "Control of Force Distribution for Power Grasp in the Digits System", Proc. IEEE Conf. on Decision and Control, pp. 1960-1965, 1990.

[34] J. C. Trinkle, J. M. Abel, and R. P. Paul, "An Investigation of Frictionless Enveloping Grasping in the Plane", Int. J. of Robotics Research, vol. 7, pp. 33-51, 1987.

[35] T. Watanabe and T. Yoshikawa, "Grasping Optimization Using a Required External Force Set", IEEE Trans. on Automation Science and Engineering, vol. 4, pp. 52-66, 2007.

[36] Y. Yin, Z. Luo, M. Svinin, and S. Hosoe, "Hybrid Control of MultiFingered Robot Hand for Dexterous Manipulation", Proc. IEEE Int. Conf. on Systems, Man and Cybernetics, pp. 3639-3644, 2003.

[37] L. Biagiotti, H. Liu, G. Hirzinger, and C. Melchiorri, "Cartesian Impedance Control for Dexterous Manipulation", Proc. IEEE Int. Conf. on Robotics and Automation, pp. 3270-3275, 2003.

[38] S. Hutchinson, G. Hager, and P. Corke , A Tutorial on Visual Servo Control", IEEE Trans. on Robotics and Automation, vol.12, pp. 651$670,1996$.

[39] D. Terzopoulos, J. C. Platt, H. Barr, and K. Fleischer, "Elastically Deformable Models", Proc. of the Anmual ACM SIGGRAPH '87 Conference, vol. 21. pp. 205-214, 1987.

[40] L. Biagiotti, F. Lotti, C. Melchiorri, and G. Vassura, How Far is the Human Hand: A Review on Anthropomorphic Robotic End-effectors, University of Bologna, Italy, 2004.

[41] D. Alba, M. Armad, and R. Ponticelli, "An Introductory Revision to Humanoid Robot Hands", in Climbing and Walking Robots, Springer Berlin Heidelberg, 2005.

[42] ***, Shadow Dexterous Hand C5, http://www.shadowrobot.com.

[43] ***, DLR Hand 2, http://www.dlr.de/rm-new/en.

[44] ***, Barrett BH8 Robotic Hand, http:/www.barrett.com/robot.

[45] M.C. Carrozza, F. Vecchi, S. Roccella, L. Barboni, E. Cavallaro, S. Micera, and P. Dario, "The ADAH Project: An Astronaut Dexterous Artificial Hand to Restore the Manipulation Abilities of the Astronaut", Proc. 7th ESA Workshop on Advanced Space Technologies for Robotics and Automation, 2002.

[46] S. Thrun, W. Burgard, and Fox D., Probabilistic Robotics, The MIT Press, 2005.

[47] B. Abou Merhy, P. Payeur, and E.M. Petriu, "Texture-Based Segmentation of 3D Probabilistic Occupancy Maps for Robot Navigation", Proc. 3rd Int. Conf. on Autonomous Robots and Agents, pp. 231-236, 2006.

[48] P. Payeur, C. Pasca, A.-M. Cretu, and E.M. Petriu, "Intelligent Haptic Sensor System for Robotic Manipulation", IEEE Trans. on Instrumentation and Measurement, vol. 54, pp. 1583-1592, 2005. 